\title{
El estudio de las traducciones de Beijing Coma en español, inglés y chino
}

\author{
Menghsuan Ku \\ Universidad Nacional de Chengchi \\ elenaku@nccu.edu.tw
}

Recibido: 4 de octubre de 2013

Aceptado: 25 de enero de 2014

\section{RESUMEN}

Beijing Coma es una novela larga que describe el movimiento del 4 de junio a través del recuerdo de un chico, Dai Wei, que quedó en coma a consecuencia de un tiro el día 4 de junio de 1989 en la Plaza Tiananmen. Después de diez años, Dai despierta, pero se encuentra preso en un cuerpo paralizado. El mundo exterior ha cambiado, la memoria del pueblo ha sido borrada por el gobierno, solo los que estaban en coma se hallan a salvo. La motivación para contrastar la traducción al español con el inglés y con la versión en chino, reside en estudiar la ideología de los traductores, y las estrategias que escogen. La importancia y el valor de nuestro trabajo vienen dados por su carácter empírico y pionero, puesto que en este campo de los estudios castellano-chino faltan suficientes análisis de traducciones como para observar una tendencia objetiva y principal.

Palabras clave: traducción, empírico, ideología, estrategia, chino.

\section{A Comparison of the Spanish and English translation of the Chinese novel Beijing Coma}

\begin{abstract}
Beijing Coma is a novel, in which the protagonist Dai Wei tells the Tiananmen events through his memories. After being shot in the Tiananmen Square on June 4, 1989, Dai Wei fell into a coma. Ten years later, he awakes, however, in a paralyzed body, discovering that the world outside has changed and that the citizens' memories, except of a "vegetable", have been erased by the government. This study compares the Spanish and English versions of the Chinese novel, with the intention to explore the ideology and translation strategies used by the translators. Since there has been few translation analysis based on the Chinese and Spanish language pairs, this research is expected to provide empirical and pioneering values to observing the objective and mainstream trend in translation.
\end{abstract}


Keywords: translation, empirical, ideology, strategy, Chinese.

Sumario: 1. Introducción. 2. Ma Jian, sus obras y traducciones al español. 2.1. Ma Jian, un escritor chino exiliado. 2.2. Las obras de Ma Jian traducidas al español. 3. Análisis de los TMs: ejemplos de los TMs que no coinciden con el TO. 3.1. La amplificación y la especificación favorecen la comprensión. 3.2. Errores que pasan de la versión inglesa a la traducción española. 4. Conclusiones.

\section{Introducción}

El inicio de la traducción de obras chinas al español se puede remontar al siglo XVI, cuando la obra Espejo Rico del Claro Corazón (Beng Sim Po Cam) fue traducida al español por Fr. Juan Cobo, O. P. Aunque la primera traducción se realizó hace mucho tiempo, no por ello podemos hablar del inicio de un movimiento de traducciones de obras en chino. De hecho, en la actualidad, las obras chinas traducidas al español no ocupan un mercado demasiado llamativo, y debido al poco interés que hay, para reducir el presupuesto, la mayoría de las novelas chinas son traducciones indirectas del inglés, aunque no todas ellas aluden claramente al texto original en la página de créditos. Por tanto, es fácil confundir a los lectores que no perciben que son traducciones indirectas camufladas.

Entre las novelas chinas modernas traducidas al español, hemos fijado nuestra atención en Pekín en coma, debido a su extensión, de más de 650 páginas. Nos llama la atención el hecho que se trata de una historia real que ocurrió no hace más de medio siglo en China y que está narrrada de manera dinámica, de modo que los lectores no pierdan el interés y la lean con facilidad. Los textos que estudiaremos son la novela en chino, 肉之地 [Pekín en coma], la traducción del chino al inglés de Flora Drew, Beijing Coma y la traducción al español, Pekín en coma, a partir del inglés, de Jordi Fibla,. Por un lado, puesto que la traducción al inglés fue realizada por la pareja sentimental del autor, consideramos que debía de ser bastante fiel a la novela china. Por otro lado, suponemos que Flora Drew tiene un nivel alto de chino y que por ello no habrá desviaciones básicas.

Respecto a la estructura de este trabajo, en primer lugar ofrecemos información sobre Ma Jian, el autor exiliado chino, luego sobre sus obras y las obras traducidas al español. En el tercer apartado, ofrecemos ejemplos detectados de oraciones que

\footnotetext{
1 El título chino de la versión en el mercado taiwanés es 肉之土, literalmente Tierra de carne, publicado por la editorial Asianculture (允晨) mientras que la versión en Hong Kong se llama 北京植物人, Pekín en coma. En chino se usan ambos nombres, aunque el primero suena más fílosófico y no es tan claro como el segundo. De hecho, el título de la publicación hongkonesa se corresponde con el título en inglés y en español y permite que el libro se identifique rápidamente. Consideramos que la causa por la que se escogen diferentes nombres para una misma novela en chino proviene de la influencia occidental. Sabemos que Hong Kong fue colonia inglesa hasta 1997 y se ha convertido en un territorio internacional. Taiwán, por otro lado, conserva relativamente más intacta la cultura china. Por tanto, no es de extrañar que la editorial taiwanesa se decante por el nombre literario y la hongkonesa por el más coloquial.
} 
muestran la discrepancia entre el texto original y las traducciones, así como unas erratas que hemos observado. No obstante, apreciamos el derecho del traductor a traducir con libertad; de hecho, hemos observado muchas veces que no es necesario que las traducciones se correspondan con las palabras exactas del texto original sino que expresen la idea original y que puedan ser entendidas por los lectores. En la bibliografía, incluimos los libros que hemos tomado como referencia y una gran cantidad de recursos en línea, ya que hay pocos estudios disponibles sobre las obras de Ma Jian. Esto es debido en parte a la censura de sus obras y al hecho de que su vida y su postura crítica despiertan el interés de los medios por conocer más acerca del escritor y su obra.

\section{Ma Jian, sus obras y traducciones al español}

En este apartado presentamos al autor de la obra que estudiamos, Ma Jian, sus novelas publicadas y traducidas al castellano. Es una pena que a pesar de que sus obras se hayan traducido a muchas lenguas, no ha conseguido publicar en su patria, China, escenario de los acontecimientos principales de sus obras, debido a la censura.

\subsection{Ma Jian, un escritor chino exiliado}

Ma Jian (1953- ) es un escritor chino exiliado en Londres en 1999 por motivos políticos, por lo que lleva más de veinte año viviendo en el extranjero. En China ejerció el periodismo y posteriormente se dedicó a escribir novelas. En 2009 recibió el premio Índice de la Censura (Index on Censorship) y el Premio a la Libertad de Expresión (Freedom of Expression Award), cuando se cumplían justo veinte años del Movimiento 4 de junio y también al año siguiente de la publicación de la novela Pekín en coma, la novela que precisamente describe el alzamiento en 1989 de los alumnos chinos poniendo en juego sus propias vidas en la Plaza Tienanmen en contra de la elevada presión del gobierno comunista. Ma Jian pasó un mes entero en la Plaza Tiananmen, siendo uno de los testigos de la manifestación. A finales de mayo, Ma Jian regresó a Qingdao a visitar a su hermano mayor, quien se hallaba en coma debido a una caída. Partiendo de ese incidente el escritor reflexionó sobre la idea de que el gobierno quería limpiar la memoria de todos, tanto la parte material como la espiritual, pero las personas en coma sobreviven debido a que su cuerpo inactivo protege su memoria.

Según el autor de la novela, Ma Jian, "una novela puede revelar solo una perspectiva de la historia aunque la historia pueda verse desde diferentes ángulos" (DWnews 200). Esta novela nos muestra la historia desde el punto de vista del protagonista, Dai Wei, y cómo la perspectiva del protagonista cambia al despertar del coma. Dai Wei es un estudiante universitario, que se queda en coma al recibir un balazo en la plaza. Cuando despierta después de diez años en coma, Dai Wai se da cuenta de que en realidad él es el único que está vivo, ya que los otros supervivien- 
tes sí que parecen estar en coma. La idea central de la novela refleja la tensión entre la propia individualidad y el interés colectivo; esto se refleja en varios fragmentos donde se vislumbra que el pueblo siempre tiene que sacrificarse para acomodarse a las órdenes gubernamentales.

Ma Jian ha confesado que lo que deseaba expresar en este libro de Pekín en coma es la necesidad de "encontrar la dignidad de la vida" (Lin 2010). Debido al contenido de la novela, Pekín en Coma nunca ha podido publicarse en China aunque ya tenemos una versión en chino no simplificado en Taiwán. En 2008 se publicó su versión inglesa ${ }^{2}$ y posteriormente las versiones de otras lenguas como español, francés, italiano, etc. De hecho, ha sido traducida a más de veinte lenguas extranjeras.

Entre las obras publicadas por Ma Jian, la primera y por la que se hizo famoso fue Saca la lengua 亮出你的吉苔或空空蕩蕩, que vio la luz en los años ochenta, auque recomendada por el primer Premio Nobel literario chino, Gao Xingjian 高行健, no pudo escapar a la censura. Incluso el editor general Liu Xinwu 劉心武fue despedido de la Editorial Literatura del Pueblo. El autor escribe principalmente novelas largas, y de momento ya tiene una variedad de publicaciones, a saber: Pensando perplejo思惑, El escritor, las mujeres y el partido 拉麵者, Nueve bifurcaciones九條叉路, Polvo rojo 紅鹿/非法流浪 ${ }^{3}$, Pekín en coma肉之土, etc. La última tardó diez años en terminarla. Debido a su tono sarcástico y al contenido exótico de sus obras para los occidentales, ha cosechado bastante éxito.

\subsection{Las obras de Ma Jian traducidas al español}

Ma Jian comenzó su vida de escritor desde la libertad del exilio y gracias a las traducciones al inglés, los lectores de otras lenguas tienen la oportunidad de disfrutarlas a través de traducciones indirectas. Las cuatro novelas de Ma Jian traducidas al español son traducciones del inglés de Flora Drew, publicadas en 2006, 2007 y 2008. A continuación desglosamos las obras de Ma Jian traducidas al español. Podemos observar que la difusión de la literatura china moderna depende bastante del círculo sinólogo inglés y el círculo español todavía lleva unos años de retraso respecto al anglonsajón.

\section{Polvo Rojo [紅鹿/ 非法流浪]}

Editorial: Planeta (352 páginas)

Publicación: 2006

Al inicio de los años ochenta, Ma Jian trabajaba como periodista oficial y estaba bajo la vigilancia de la policía. Cansado de soportar el control exigente e inhumano al que estaba sometido, y de que la policía lo detuviera o llamara con fre-

\footnotetext{
2 Para información detallada de Beijing Coma, veáse la bibliografía de este trabajo.

3 Hemos observado que en las obras de Ma Jian las páginas de créditos ponen 紅塵 como el nombre chino de Red Dust. Sin embargo, en la versión publicada en chino el nombre es 非法流浪. Consideramos que es más completo colocar ambos nombres en nuestro trabajo.
} 
cuencia, decidió marcharse de Pekín. Polvo rojo es una novela de viajes que describe el viaje de Ma Jian a pie del norte al sur, del centro al noroeste, del noroeste al Tíbet. Durante estos tres años de vagabundear, Ma Jian trabajó como peluquero, adivino, etc. también se perdió en el desierto y pasó tres días sin beber. A través de esta novela, más allá de describir China, Ma Jian describe el sistema autocrático de China ${ }^{4}$. Gracias a la traducción de Polvo rojo en inglés, Red Dust, en 2002 se le otorgó el Premio de Libros de Viajes de Thomas Cook, siendo el primer escritor chino en ganar este premio literario inglés de máximo prestigio en literatura de viajes.

\section{Saca la lengua [亮出你的舌苔或空空蕩蕩]}

Editorial: Emecé (112 páginas)

Publicación: 2006

La segunda obra de Ma Jian traducida al español es Saca la lengua, aunque en 2002 ya habían publicado la versión en catalán, traducida por Sara Rovira Esteva. Se trata de una novela de longitud media y estilo surrealista que desarrolla temas como el amor, la civilización, la empatía, la ética y la religión. Fue publicada en el año 87 cuando el Movimiento Estudiantil democrático falló y el gobierno buscó a toda costa pruebas y archivos sospechosos a favor de la liberalización de la clase burguesa. Saca la lengua fue publicada en la Revista de literatura del pueblo人民文學 y unos días después de la publicación, el gobierno chino la censuró y la destruyó. Veinte años después, se publicó de nuevo, pero esta vez en inglés. La traducción le abrió una puerta hacia el mundo anglosajón, en 2007 fue finalista del premio literario americano Kiriyama junto con otros cuatro escritores.

\section{El escritor, las mujeres y el partido [拉麵者]}

Editorial: Emecé (240 páginas)

Publicación: 2007

El escritor, las mujeres y el partido fue traducida como The Noodle Maker en inglés, describiendo conversaciones entre un escritor y un donante de sangre que recuerdan un pasado poco agradable y ridículo. Según la entrevista a Ma Jian (Shi, Tang 2004), la imagen del maestro de fideos es la de la dictadura porque con intención deforma la ideología del pueblo. Para Ma Jian, Mao y el partido comunista son el maestro de fideos, mientras que los chinos son como una masa que ha sido manejada y formada a su antojo. En la misma entrevista, indica el autor que "la gente que vive en la dictadura no cree que tenga ningún problema ni se da cuenta siquiera de que haya algún problema [...]. Viven con el miedo y están tan acostumbrados a él que ni siquiera lo reconocen." Esta novela consiguió al final ser publicada, aunque el nombre del autor y el contenido sufrieron modificaciones. Con todo, el gobierno amenazó a la editorial y la novela dejó de venderse en el mercado. La traducción en inglés ganó en 2006 el segundo lugar del premio de Pen Translation Prize.

\footnotetext{
${ }^{4}$ En la introducción de esta novela, Gao Xingjian nos recuerda que "si a un escritor se le permite viajar solo por su tierra, es una limitación social. Por suerte Ma Jian ha asumido su responsabilidad y ha transmitido a través de sus novelas toda la riqueza de la experiencia de sus viajes". (Ma 2006: 21).
} 


\section{Pekín en coma [北京植物人]}

Editorial: Mondadori (658 páginas)

Publicación: 2008

La última novela publicada en castellano es Pekín en coma, novela satírica que refleja el movimiento estudiantil en la Plaza Tiananmen en el año 89. Contituyó un trabajo de diez años para Ma Jian, el autor ha creado el personaje del protagonista Dai Wei quien sufrió un balazo en Tiananmen quedando en coma y el de su madre, un miembro leal del partido comunista, que le cuidó durante más de una década. Un día cuando Dai Wei despierta del coma, descubre que todo el mundo parece estar en coma con un cuerpo sin recuerdos, menos él que por estar en coma ha tenido la suerte de poder guardar el pasado. Hasta la fecha, el gobierno de China prohíbe cualquiera información acerca de aquel movimiento del año 89, con la intención de borrar los fragmentos del pasado.

\section{Análisis de los TMs: ejemplos de los TMs que no coinciden con el TO}

La traducción de Pekín en coma, según Shuttleworth \& Cowie (2004: 76) es una traducción indirecta porque el texto no se traduce directamente del texto original sino a través de la versión de otra lengua. Toury ha indicado que la traducción indirecta no debe evitarse como una enfermedad, es más bien un síndrome por descubrir, ya que hay que plantearse los factores que influyen en la traducción indirecta (1995: 129). Por su parte, Ringmar (2004) menciona las relaciones internacionales como uno de los factores que inciden en la traducción, ya que las culturas más poderosas juegan un papel importante a la hora de promover traducciones y convertirse en textos intermedios. Basándonos en estas teorías ya estudiadas, en este trabajo preferimos estudiar los textos en sí, concretamente las relaciones entre la novela original y las traducciones en lugar de los factores exteriores. Estamos de acuerdo con Lu Xun (2005: 532) en cuanto a la valoración positiva de la calidad de la traducción indirecta, porque "una traducción indirecta realizada por traductores con profundo conocimiento de la lengua original a veces es mejor que una traducción directa realizada por un traductor leal sin suficiente conocimiento del texto original."

Para estudiar los textos meta en inglés y en castellano, aplicamos las técnicas de traducción (Hurtado 2001) como instrumento. La traductora ha adoptado varias técnicas a lo largo del proceso de traducción de esta larga novela. Consideramos que los elementos lingüísticos culturales son aspectos idóneos para justificar las estrategias de la traducción. A través de un contraste de tres textos, hemos visto que en la traducción se opta por diferentes técnicas aleatoriamente. En los siguientes dos ejemplos la traductora se ha decantado por técnicas distintas: en el Ejemplo 1, encontramos una traducción literal; en el Ejemplo 2, se ha suprimido esta referencia cultural china, que significa que en ocasiones no hay más remedio que rebelarse para poder resistir. A lo largo de nuestro trabajo, adoptamos la sigla TO para el texto original en chino, TMing. para el texto meta en inglés y TMesp. para el texto meta español. 
Ejemplo 1

TO 行啊, 癩蛤嘛吃了天㧶肉。(p. 452) [El sapo perezoso se atreve a probar la carne del cisne]

TMing. I see. "The lazy toad dares taste the meat of the swan." (p. 515)

TMesp. Comprendo. ¡«El sapo perezoso se atreve a probar la carne del cisne»! (p. 487)

Ejemplo 2

TO 「是逼上梁山了, 哥們。我有預感, 不成功便成仁...」。(p.452) [se ve obligado a rebelarse]

TMing. Ø I just have a gut feeling that if we don’t do something dramatic now, our movement will collapse. (p. 515)

TMesp. Ø Intuyo si no hacemos ahora algo espectacular, nuestro movimiento se vendrá abajo. (p. 487)

Tal y como hemos mencionado, vemos que en las diversas traducciones, sean fieles o no a la traducción integra del texto original y su idea, han empleado una variedad de técnicas de traducción. En los dos apartados siguientes, vamos a desglosar varios ejemplos de traducciones que no se corresponden con el texto original; con ello queremos por una parte resaltar la función de una traducción no equivalente, que aunque no se corresponda con el texto original, sí que puede explicar más en detalle el contexto utilizando la técnicas de amplificación y especificación, de modo que los lectores conozcan mejor los escenarios de la historia. Por otra parte, estudiaremos algunos errores y veremos que en la traducción indirecta se cometen los mismos errores que aparecieron en la traducción primaria, ya que si el traductor no conoce el texto original no puede cotejar la traducción con el original chino.

\subsection{La amplificación y la especificación favorecen la comprensión}

La amplificación requiere no sólo un manejo correcto de la lengua sino también un profundo conocimiento cultural. A lo largo de la narración de Beijing Coma encontramos versos líricos breves, fragmentos poéticos del El libro de las montañas y los mares 山海經 de la China antigua y frases hechas de anéctodas históricas, entre otras. Una información auxiliar ayuda a complementar la lectura evitando que la traducción quede demasiada implícita:

Ejemplo 3

TO 我想給大家念幾句李清照的絕句:生當做人傑 [... ] 至今思項羽, 不肯過江東！ (p. 569) [Li Qingzhao, cruzar el río]

TMing. I would like to read out a poem by Li Qingzhao, a female writer of the

Song Dynasty: "In life, we should be heroes among the living, $[\ldots]$ To this day we mourn Xiang $\mathrm{Yu}$,/ Who chose to stay and die rather than cross the Yangtse River!" (p. 652) 
TMesp. Quisiera leeros un poema de Li Qingzhao, una poetisa de la dinastía Song: «En la vida, deberíamos ser héroes entre los vivos. [...] Hoy seguimos 1lorando a Xiang Yu,/ ¡que prefirió morir a cruzar el río Yangtsé!» (p. 612)

Este fragmento relata cómo durante el Movimiento de 4 de junio, una de los líderes Bai Ling estaba cansada por llevar luchando contra el gobierno casi 20 días sin éxito. Su coraje derrotado coincide con el Rey Chu, Xiang Yu 項羽 (a.C.232a.C.202), cuando su tropa fue derrotada por Liu Bang 劉邦 (a.C.256- a.C.195) y se suicidió al lado de el río Wu 烏江, el curso superior del río Yangtsé 揚子江. Según este fragmento histórico, la poetisa Li Qingzhao 李清照 (1084-1155) compuso el "Poema de Verano" para alabar a Xiang Yu. Más que una traducción literal, ambas traducciones se decantan por las amplificaciones en indicar la identidad de Li como poetisa y el nombre del río Yangtsé donde prefirió suicidarse que escapar cruzándolo.

Frente a la traducción de la frase hecha, la técnica de la amplificación ayuda a captar la idea conotativa implícita para los lectores debido a la distancia cultural:

Ejemplo 4

TO 我是王八吃科砣, 鐵了心了。(p. 591) [soy la tortuga que come una pesa de plomo]

TMing. I'm like the turtle in the fable, which swallows a lead weight when someone comes to remove it from its pond. I will stay firm. (p. 679)

TMesp. Soy como esa testaruda tortuga de las fábulas que se traga una pesa de plomo cuando alguien trata de sacarla del estanque. Me mantendré firme. (p. 636)

Ni siquiera el chino nativo, al utilizar esta expresión, piensan en su lógica sino en su significado. Por ello, una traducción literal no resultaría adecuada debido a que el español no comparte la misma expresión. La amplificación de los dos textos meta tiene la función de completar la frase y remarcar el sentido de la expresión china, que significa estar decidido a algo.

Gracias a que la traductora de la versión inglesa posee un conocimiento detallado en cuanto a la localización y los edificios de las escenas mencionadas en la novela, la traducción amplificada en la versión inglesa favorece la claridad del contexto. En el siguiente ejemplo, el texto original de la novela en chino menciona que el personaje viene desde el Oeste de la plaza, pero sin especificar el lugar original. Sin embargo, en las traducciones se especifica que el camión blindado pasaba junto a la Gran Sala del Pueblo. La presentación del nombre del edificio deja un espacio a la imaginación de los lectores y además permite que los lectores reconstruyan la escena a través de la narración.

Ejemplo 5

TO 又一輛裝甲車從廣場西側馬路駛來，撞倒一個推自行車的人。(p. 581) [(viene) a la derecha de la plaza] 
TMing. We must launch a resolute counter attack... An armoured personnel carrier past the Great Hall of the People, knocking over a man pushing a bicycle. (p. 666)

TMesp. Otro transporte de personal blindado pasó a toda velocidad junto a la Gran Sala del Pueblo, derribando a un hombre que empujada una bicicleta. (p.625)

Las traducciones del siguiente ejemplo también han indicado el nombre de la Avenida Chang'an, donde se reunían los alumnos, además de la tienda de la Cruz Roja. Consideramos que la especificación de los nombres propios es el uso lingüístico occidental, por tanto aunque en la novela original suena natural elidir el nombre de la calle y la entidad internacional; sin embargo, Flora Drew tiende a traducir todos los nombres.

Ejemplo 6

TO 有幾個白衣護士往倒下去的同學那兒跑去, 我赶緊拉天衣往急救帳篷那兒 跑去 (p. 579) [Ø, la tienda de socorro]

TMing. Nurses in white coats ran past us to tend to the students lying on Chang'an Avenue. I pull Tian Yi up and tried to drag her towards the Red Cross tent, (p. 664)

TMesp. Unas enfermeras con bata blanca pasaron corriendo por nuestro lado para atender a los estudiantes que estaban tendiendo en la avenida Chang'an, Levanté a Tian Yi y traté de llevarla hacia la tienda de la Cruz Roja, (p.622)

Las traducciones del siguiente ejemplo son una muestra de la técnica de especificación porque el texto original especifica que se trata de una postal escrita en lengua extranjera pero no indica en qué lengua está escrita. Aunque a partir de las siguientes líneas del mismo párrafo, los lectores pueden adivinar que la postal está en ruso, la estrategia de adelantarlo es, por una parte, influencia de la costumbre en la lengua a que se está traduciendo y, por otra, es una cuestión de cohesión porque en las siguientes líneas se sigue hablando de este mismo tema.

Ejemplo 7

TO他不知道, 那張用外文寫的卡片早就被我燒了。(p.584) [lengua extranjera]

TMing. She never knew that the postcard with its message written in Cyrillic script was burnt by me. (p.670)

TMesp. Nunca sabrá que quemé aquella postal con su mensaje escrito en alfabeto cirílico. (p.628)

En el siguiente ejemplo de amplificación, las traducciones han añadido información de Zhao para los lectores que desconozcan la situación política de China, además de concretar que es el secretario general; así, el resultado final de la frase resulta más lógico, comprensible y coherente.

Ejemplo 8

TO 剛得到消息, 趙子陽希望我們堅持到天亮。(p.587) [Zhao Ziyang] 
TMing. We've just received some inside information that General Secretary Zhao Ziyang wants us to stay here until dawn. (p.674)

TMesp. Acabamos de recibir informes confidenciales de que el secretario general Zhao Ziyang quiere que sigamos aquí hasta el amanecer. (p.631)

En la novela original Pekín en coma no aparece la exclamación, sino una queja que se desarrolla como un enunciado normal. Las dos traducciones suenan mucho más expresivas debido a que la parte añadida expresa sentimientos y tiene la función de hacer la frase más tangible y recordar a los lectores que a continuación sigue la descripción de una sensación poco satisfactoria.

Ejemplo 9

TO …怎麼跟進了雞窩似的, 那兒是人住的房子。(p. 592) [Ø]

TMing. What a stench! It smells like a chicken hut in here. How can she bear to live like this? (p.681)

TMesp. ¡Qué peste! Aquí huele como un corral de gallinas. ¿Cómo puede vivir así? (p.638)

\subsection{Errores que pasan de la versión inglesa a la traducción española}

Hemos detectado errores en las traducciones, algunos son términos, por ejemplo traducen 蠍子[escorpión] (p.431) por locust (TMing. p.491) y saltamontes (TMesp. p.465), 屎粒子 [caca] (p.588) por drops of urine (TMing. p.509) y gotas de orina [TMesp. p.481], 虫尤[Chi You] (p.588) por Zi You (TMing. p.675) (TMesp. p.632), otros pertenecen a unidades mayores como oraciones.

El siguiente ejemplo no es rigurosamente una desviación, sino una manipulación de la traductora de la versión inglesa, aunque en inglés tampoco existe una expresión en la que se mencione veinte minutos como unidad simbólica cuando se llega tarde. En cuanto al lector español, es meramente una afirmación para expresar el hecho de que la otra persona ha llegado tarde. No conseguimos detectar la intención de la traductora al traducir "llegar tarde" por "esperar veinte minutos".

Ejemplo 10

TO你遲到了。 (p.541) [Has llegado tarde.]

TMing.Well, I've been waiting here for twenty minutes. (p.620)

TMesp. Pues te he estado esperando veinte minutos. (p.582)

El ejemplo 11 muestra un error bastante claro, porque lo que quería indicar el texto original es que el niño es demasiado pequeño para que se pueda saber su sexo solo por la apariencia. Sin embargo, las traducciones han interpretado que el niño es un varón.

Ejemplo 11

TO 孩子看上去小的分不出性別 (p.543)[Es tan pequeño, por tanto no se diferencia su sexualidad] 
TMing. I think it's a boy. (p.623)

TMesp. Creo que es un chico. (p.585)

El ejemplo 11 muestra el caso de una metáfora sarcástica para decir que la nuera de una vecina anciana sigue todas la instrucciones de la policía; tanto, que incluso es capaz de pegar el aviso policial que prohíbe practicar Falungong en la cabecera de la cama de la vecina. Así, la vecina no está contenta con su nuera y considera que es el perro guardián de la policía. Las traducciones transmiten una idea muy diferente a la del texto original, además de perder el tono metafórico y coloquial de la cultura china.

Ejemplo 12

TO 真是警察的看家狗(p. 551) [Es el perro guardián de la policía]

TMing. My family is worse than the police. (p.631)

TMesp. Mi familia es peor que la policía. (p.592)

La traducción indirecta se ve afectada inevitablemente por el texto intermedio, al margen de las erratas demostradas, en este apartado nos gustaría indicar que muchas veces los términos para objetos extranjeros son traducciones adaptadas de la lengua de la que se parte para la traducción. En el caso del presente trabajo, debido a que la versión en inglés es el texto original de la traduccion en español, si se encuentra un objeto poco común en la cultura española no tiene más remedio que adoptar su traducción en inglés y aplicarla a la costumbre lingüística española. Por ejemplo los lüdou 綠豆 (TO p.585) son similares a alubias verdes, así como lentejas. Sin embargo en inglés son mung beans (TMing. p.629), por ello traduce al español como alubias mung (TMesp. p. 629) Este fenómeno más que un error de traducción es una característica a tener en cuenta en las traducciones indirectas.

\section{Conclusiones}

Flora Drew, la traductora de la versión inglesa Beijing Coma, se decanta por las técnicas de amplificación y de especificación a lo largo de toda la novela. Los ejemplos que detectamos en la versión inglesa demuestran la tendencia de Drew a agregar información adicional para los lectores de la traducción, optando así por una traducción detallada y con abundantes aclaraciones. Tal y como hemos estudiado en el tercer apartado, muchos ejemplos que aplican estas dos técnicas resultan más claros y con mayor información, de modo que favorecen la comprensión de los lectores del español.

Aunque es cierto que las partes ampliadas se alejan de la lealtad hacia el texto original, lo que nos llama la atención es la influencia encadenada. Debido a que la versión inglesa es de hecho el texto original de la traducción en español, los errores que comete aquella traducción pasan sin duda a la versión española. En los casos de traducción indirecta, la tarea de cotejar la traducción con el texto de la lengua original es algo que suele ignorarse, pero que resulta fundamental. 
Por otra parte, nos gustaría recalcar la importancia y la influencia de la traducción inicial. Las obras de Ma Jian se han difundido por el mundo gracias a la traducción inglesa y a partir de esta se han realizado las traducciones a otras lenguas. Gracias a esta difusión se ha ayudado a un escritor, una acción necesaria teniendo en cuenta que se halla exiliado, no solo a expresar sus sentimientos personales, sino también a cumplir con la responsabilidad de revelar cómo es la patria de donde uno proviene, a través de la descripción de cualquier aspecto de la misma.

\section{Referencias bibliográficas}

HuRTAdo, Amparo, Traducción y traductología. Madrid: Cátedra 2001.

Lu, Xun, Obras completas de Lu Xun (Vol. 5) 魯迅全集 (第五卷). Beijing: Literatura del pueblo 人民文學 2005.

MA, Jian, Treu la llengua saburrosa. Andorra la Vella: Límits 2002. (Traducción, prólogo y notas de Sara Rovira)

MA, Jian, Polvo rojo 非法流浪. Taipei: Cite城邦 2003.

MA, Jian, Polvo rojo. Madrid: Planeta 2006.

MA, Jian, Saca la lengua. Buenos Aires: Emecé 2006. (Traducción de Alcira Calascibetta)

MA, Jian, El escritor, las mujeres y el partido. Madrid: Emecé 2007. (Traducción de María Martoccia)

MA, Jian, Beijing coma. New York: Picador 2008. (Traducción de Flora Drew)

MA, Jian, Pekín en coma. Madrid: Mondadori 2008. (Traducción de Jordi Fibla)

MA, Jian, Pekín en coma北京植物人. Hong Kong: Mirror Books明鏡 2009.

MA, Jian, Pekín en coma肉之土. Taipei: Asianculture 允晨 2010.

Shuttleworth, Mark / Cowie, Moira, Dictionary of Translation Studies. Beijing: Foreign Language and Research 外研社 2004 .

Toury, G., Descriptive Translation Studies and Beyond. Amsterdam: John Benjamin 1995.

\section{Recursos electrónicos}

DwNEWS, El equipo de redacción (ed.), 《多維新聞. 遺忘與昏迷. 馬建談長篇小說 《北京植物人》2009-8-29 [DWNEWS.COM. Olvido y en coma. Ma Jian habla de su novela larga Pekín en coma]», en línea: consultado en mayo de 2013.

Disponible en: <http://global.dwnews.com/news/2009-08-29/5077304.html>

IFEX. «Índice de la censura homenaje a defensores de libre expresión», en línea: consultado en mayo de 2013.

http://www.ifex.org/awards/2009/04/22/index_on_censorship_2009/es/

LIN, Xinyi 林欣誼, 《作家專訪: 馬建《肉之王》無能肉身生命回溯 2010-6-18 [Entrevista al autor: Ma Jian. Pekín en coma: un recuerdo de la vida en un cuerpo inútil]», en línea: consultado mayo de 2013. Disponible en: <http://news.chinatimes.com/>

Prado-FonTs, Carles, «¿El fin de la Gran Muralla?»Revista de Libros. 177 (2011), en línea: consultado en mayo de 2013. Disponible en: http:/www.revistadelibros.com/articulos/ el-fin-de-la-gran-muralla

RINGMAR, M., “"Roundabout Routes”. Some Remarks on Indirect Translation» en: MUS, F. (ed.), Selected papers of the CETRA Research Seminar in Translation Studies 2006., en 
línea: consultado en mayo de 2013. Disponible en: <http:/www2.arts.kuleuven.be/info/ bestanden-div/RINGMAR.pdf>

SHI, Dongni / TANG, Ying 史東妮, 唐英, 《旅英作家馬建談《拉麵者》在中國 2004-8-3 [El escritor exiliado en Inglaterra Ma Jian habla de The noodle maker en China] », en línea: consultado en mayo de 2013. Disponible en: <http://www.epochtimes.com/b5/4/ 8/3/n615330.htm>

WAN, Yizhong 萬毅忠, 《多維專訪馬建：我們使用的漢語沒有歷史 2007-6-12 [DWNEWS.COM. entrevista a Ma Jian: el chino que usamos no tiene historia]», en línea: consultado en mayo de 2013. Disponible en: <http://politics.dwnews.com/big5/ news/2007-06-12/3006889-2.html> 